\title{
ADESÃO AO TRATAMENTO ANTIRRETROVIRAL: VIVÊNCIAS DE ESCOLARES
}

\author{
Sueny da Silva Martins ${ }^{1}$, Tathiana Silva de Souza Martins ${ }^{2}$
}

\footnotetext{
${ }^{1}$ Especialista em Enfermagem Pediátrica. Enfermeira da Unidade de Terapia Intensiva Pediátrica do Hospital Cardoso Fontes - MS. Rio de Janeiro, Brasil. E-mail: suenymartins@yahoo.com.br

${ }^{2}$ Mestre em Enfermagem. Enfermeira da Unidade Pediátrica do Hospital Universitário Pedro Ernesto da Universidade do Estado do Rio de Janeiro. Enfermeira da Gerência de Risco do Instituto Nacional de Traumatologia e Ortopedia - MS. Rio de Janeiro, Brasil. E-mail: tathinurse@gmail.com
}

RESUMO: Pesquisa de abordagem qualitativa, cujos objetivos foram descrever o processo de adesão através da análise do cotidiano dos escolares e do processo de ocultamento do HIV/aids e identificar situações vivenciadas pelos escolares que possam influenciar na adesão à Terapia Antirretroviral. Utilizou-se como técnica para coleta de dados a entrevista estruturada, direcionada a escolares em uso de terapia antirretroviral e acompanhados desde o diagnóstico da aids no ambulatório pediátrico de doenças infectoparasitárias. Os relatos dos escolares foram analisados à luz da análise de conteúdo e agrupados nas seguintes categorias: o cotidiano dos escolares no processo de adesão à terapia antirretroviral e o silêncio na constituição de um cotidiano trágico. É preciso que os profissionais de saúde reconheçam que a não adesão é um fenômeno que deve ser trabalhado junto à criança e que é importante a implementação de estratégias capazes de incentivar a adesão ao tratamento.

DESCRITORES: Agentes antirretrovirais. Enfermagem pediátrica. Síndrome de Inmunodeficiencia Adquirida. Criança.

\section{ADHERENCE TO ANTIRRETROVIRAL THERAPY: EXPERIENCE WITH SCHOLARS}

\begin{abstract}
The objectives of this qualitative study were to describe the adhering process through analyzing the daily lives of scholars and the process of hiding one's HIV/Aids, as well as to identify situations they have lived which may influence their adherence to Antiretroviral Therapy. The data was collected through structured interviews given to students receiving antiretroviral therapy and accompanied from their HIV diagnosis in a pediatric infectious and parasitic disease clinic. The participants' reports were analyzed according to content analysis and grouped into the following categories: student daily life in the process of adhering to antiretroviral therapy, and silence in constructing a tragic daily life. It is necessary that health care professionals recognize that non-adherence is a phenomenon which should be dealt with together with the child and that it is important to implement strategies capable of offering incentive to adhere to treatment.
\end{abstract}

DESCRIPTORS: Anti-retroviral agents. Pediatric nursing. Acquired Immunodeficiency Syndrome. Child.

\section{ADHESIÓN AL TRATAMIENTO ANTIRRETROVIRAL: EXPERIENCIA DE ESCOLARES}

RESUMEN: Se trata de una investigación cualitativa que tuvo como objetivos: describir el proceso de adhesión al tratamiento antirretroviral por medio del análisis de la vida cotidiana de los estudiantes y el proceso de ocultación del VIH/Sida, e identificar situaciones que puedan influir en la adhesión al tratamiento antirretroviral. Como herramienta para la recolección de los datos se utilizó la entrevista estructurada, dirigida a los escolares que usan antirretrovirales y son seguidos desde el momento del diagnóstico del Sida en la clínica de enfermedades infecciosas y parasitarias. Los informes de los estudiantes se analizaron con base en el análisis de contenido y se agruparon en las siguientes categorías: la vida cotidiana de los estudiantes en el proceso de adhesión al tratamiento antirretroviral y el silencio en la formación de una tragedia cotidiana. Se necesita que los profesionales de la salud reconozcan que la falta de adhesión es un fenómeno que debe ser tratado con el niño y que es importante implementar estrategias para fomentar la adhesión al tratamiento.

DESCRIPTORES: Agentes anti-retrovirales. Enfermería pediátrica. Síndrome de Inmunodeficiencia Adquirida. Niño. 


\section{INTRODUÇÃO}

O presente estudo teve como objeto de investigação a adesão de escolares (6-11 anos) vivendo com Vírus da Imunodeficiência Humana (HIV)/ Síndrome da imunodeficiência adquirida (aids) à Terapia Antirretroviral (TARV).

A epidemia do HIV/aids é realidade mundial e configura um dos mais sérios problemas contemporâneos no contexto da saúde pública. No Brasil, desde o início da década de 80 , até junho de 2009, foram notificados ao Ministério da Saúde (MS) 462.237 casos de aids. Desse total, $188.396(40,75 \%)$ referem-se a adultos do sexo feminino e $16.071(3,5 \%)$ a crianças menores de 13 anos de idade. Esses resultados confirmam a atual situação da epidemia no Brasil, caracterizada pela heterossexualização, feminização e aumento da transmissão vertical. ${ }^{1}$

Com o crescimento do número de casos de aids entre mulheres em idade fértil e o consequente aumento do risco de transmissão vertical do HIV, constata-se aumento de crianças e recém-nascidos afetados pelo referido vírus. ${ }^{2}$

No entanto, o advento de medicamentos Antiretrovirais (ARVs) tem repercutido positivamente nas condições de vida e saúde de crianças e adolescentes vivendo com HIV/aids, permitindo ganhos no processo de desenvolvimento e na qualidade de vida. Ao contrário do que ocorria no início da epidemia da aids, atualmente um grande número de crianças infectadas por meio da transmissão vertical do HIV durante a gestação, parto ou aleitamento é capaz de atingir a adolescência e a idade adulta. ${ }^{3}$

O surgimento de novos medicamentos e a ampliação da disponibilidade dos ARVs resultou na melhoria significativa na qualidade de vida dos indivíduos infectados pelo HIV. Tal fato contribuiu para transformação da aids em uma condição crônica, ou seja, doenças que são permanentes, e que cursam com incapacidade leve, causadas por alterações patológicas irreversíveis, exigindo treinamento especial do paciente para reabilitação ou um longo período de supervisão, observação e cuidado. ${ }^{4}$

Sabe-se que a efetividade do tratamento de uma condição crônica é determinada pela eficácia do medicamento, extensão da adesão, bem como pelas características do doente e da doença. A taxa de adesão para tratamento de doenças crônicas é em geral baixa, o que agrava consideravelmente a eficácia da terapêutica. A baixa aderência é um dos problemas mais graves do sistema de assistência à saúde, tornando ineficaz o melhor tratamento disponível. ${ }^{4}$

Entende-se que a adesão ao tratamento antirretroviral resulta em uma vida com melhor qualidade, uma vez que, ao estar melhor, o indivíduo tem a possibilidade de viver não mais com as marcas imputadas pela doença, como nos anos 80 , quando se reconhecia os "aidéticos" por sua aparência.

Nesse contexto, a criança que convive com aids tem direito a viver sua infância como criança, não sendo percebida e tratada somente como um doente que necessita de cuidados, mas como ser que precisa chorar, brincar, sonhar, estudar, relacionar-se, enfim, ser-criança. Percebe-se que é imprescindível reconhecer o ser humano na integralidade de sua existência, respeitando suas vivências, experiências e sentimentos, mostrandose disponível à relação de ajuda para compartilhar momentos singulares do encontro de cuidado, buscando possibilitar a qualidade de vida, por meio de escolhas compartilhadas, livres e responsáveis, e que são estas atitudes humanas que configuram a ética solidária do cuidado em enfermagem. ${ }^{5}$

Fica claro que a adesão é um desafio diário, e a possibilidade de incentivar o portador da condição crônica a optar pela saúde é um dos objetivos da equipe de saúde. E não há como atingir esse objetivo de forma ampla sem um programa estruturado de adesão e por esse motivo, todas as iniciativas destinadas ao desenvolvimento desse tema devem ser implementadas.

Desta forma, a enfermagem, como participante da equipe multidisciplinar dos serviços de apoio e atendimento aos clientes com aids, deve contribuir para melhorar a conduta do sujeito no cuidado à sua saúde, auxiliando-o a recorrer a técnicas que o ajudem a desenvolver e consolidar a sua capacidade de autocuidado.

Aliando o contexto da aids pediátrica ao dia a dia na consulta de enfermagem, em um Ambulatório Pediátrico de Doenças Infecto Parasitárias (DIP) no município do Rio de Janeiro, em 2009, percebeuse aspectos no cotidiano dos escolares vivendo com aids que influenciavam na adesão e não-adesão à TARV. O enfermeiro, a partir dos problemas identificados precisava desenvolver estratégias que visassem à adesão da referida clientela.

No período classificado como escolar a criança está dirigida para fora do grupo familiar, e está em contínuo avanço nos desenvolvimentos físico, mental e social, além da aquisição de habilidades 
e competências. ${ }^{6}$ Espera-se que os enfermeiros pediatras possam a partir deste estudo prestar um cuidado integralizado fortalecendo e aprimorando a capacidade de tomada de decisão do escolar favorecendo à adesão, a qual possibilitará uma melhor qualidade de vida da criança, gerando a diminuição de risco de cepas resistentes e a transmissão do HIV.

Esta pesquisa tem como objetivo descrever o processo de adesão através da análise do cotidiano dos escolares e do processo de ocultamento do HIV/aids e identificar situações vivenciadas pelos escolares que influenciam no processo de adesão à TARV.

\section{PERCURSO METODOLÓGICO}

Trata-se de uma pesquisa de campo do tipo exploratório-descritiva e com abordagem metodológica qualitativa. A escolha por este tipo de estudo adequou-se na medida em que a identificação de possíveis problemas de adesão dos escolares vivendo com HIV/aids à TARV pode ser melhor compreendida a partir da aquisição dos relatos dessas crianças, do que pela construção de um modelo teórico a partir de uma revisão bibliográfica. ${ }^{7}$

Para melhor orientação no desenvolvimento do estudo foram formuladas as seguintes questões norteadoras: a) Como é a adesão à terapia antirretroviral dos escolares que vivem com $\mathrm{HIV}$ / aids em acompanhamento em ambulatório de DIP Infantil de um Hospital Universitário localizado no município do Rio de Janeiro ?; e b) Quais situações são vivenciadas pelos referidos escolares que podem vir a influenciar na adesão à terapia antirretroviral?

Mediante tais questões o cenário escolhido para realização da pesquisa foi Serviço de DIP do Ambulatório de Pediatria de um Hospital Universitário localizado no município do Rio de Janeiro. Esta unidade dispõe de duas salas ambulatoriais e realiza semanalmente consultas a pacientes na faixa etária de zero a 18 anos soropositivos e expostos ao HIV. A equipe multiprofissional conta com infectologistas pediátricos, enfermeiros, assistente social, nutricionista e psicólogo. Durante o atendimento, além dos aspectos referentes à aids, são abordados também o crescimento ponderoestatural, resultados de exames laboratoriais, pareceres de outras especialidades, a história vacinal, além do estímulo da adesão à terapêutica medicamentosa.

Constituíram critérios de inclusão para seleção dos sujeitos, idade, diagnóstico de HIV/ aids desde a infância, estar em uso de TARV, fazer acompanhamento no ambulatório de DIP Infantil desde o diagnóstico da doença e a assinatura do Termo de Consentimento Livre e Esclarecido (TCLE) pelo responsável legal.

Foram selecionados 10 sujeitos participantes com idades entre seis e 11 anos, de ambos os sexos (seis meninas e quatro meninos), todos devidamente matriculados e cursando o ensino fundamental. Optou-se pela faixa etária escolar, pois se sabe que é por volta dos seis aos onze anos de idade, que a criança é capaz de operar o pensamento concreto, estendendo-o à compreensão do outro e às consequências de boa parte dos seus atos. ${ }^{8}$

A coleta das informações ocorreu entre maio e julho de 2009, através da entrevista estruturada que incluía duas etapas. A primeira etapa abordou os dados de identificação da criança e, a segunda, as questões diretivas sobre a vivência com a doença e rotina diária de cada escolar.

A opção pela entrevista ocorreu por possibilitar acesso a dados descritivos da linguagem do sujeito a partir dos objetivos do estudo e por permitir que o investigador desenvolva uma idéia sobre a forma como estes sujeitos interpretam aspectos do mundo.

A entrevista foi realizada com cada criança, individualmente, após a assinatura do TCLE pelo responsável legal das mesmas, permitindo a gravação dos relatos em fita K7.É importante lembrar que as entrevistas foram previamente agendadas com as crianças e realizadas em local reservado no próprio ambulatório de DIP.

O projeto de pesquisa foi avaliado pelo Comitê de Ética em Pesquisa com Seres Humanos do Hospital Universitário Pedro Ernesto, que está vinculado à Universidade do Estado do Rio de Janeiro, sendo aprovado sob o protocolo $\mathrm{n}^{\mathrm{o}} 2350$. Também foram assegurados aos participantes, por meio do TCLE, o anonimato de suas identidades, o esclarecimento de dúvidas pertinentes ao estudo, bem como a autonomia e voluntariedade na participação da pesquisa. Após a leitura deste documento pela pesquisadora, o mesmo foi assinado em duas vias de igual teor pelas responsáveis legais dos participantes e pela pesquisadora. $\mathrm{Na}$ apresentação dos resultados os depoentes estão identificados por nomes de super-heróis.

A análise dos dados qualitativos foi realizada por meio de análise de conteúdo, com o objetivo de permitir o acesso aos diversos conteúdos, explícitos ou não, na comunicação verbal do cotidiano dos escolares com HIV/aids, avaliando o tipo de fala e a interpretação que se pretendia como ob- 
jetivos. ${ }^{9}$ Essa técnica prevê algumas etapas para a análise dos relatos, que foram seguidas após o processo de transcrição das fitas. Assim, executou-se os seguintes passos, para a análise e interpretação dos dados coletados:

- pré-análise: efetuou-se a leitura flutuante, que se constitui por leitura exaustiva das entrevistas, buscando impregnação dos sentidos das respostas emitidas pelos escolares às questões norteadoras, respondidas pelos dez sujeitos investigados, garantindo a regra de representatividade e assegurando a pertinência da fonte de informação e do objetivo de análise;

- exploração do material: onde os relatos foram agrupados e mapeados e, finalmente, foi realizada a releitura dos relatos, visando reorganizá-los para a elaboração das categorias finais, que foram submetidas a um processo de discussão à luz da literatura pertinente, levando em conta os conteúdos comuns; posteriormente, encontraramse as unidades temáticas pertinentes.

A partir da análise, os dados foram organizados, segundo suas unidades de significação, nas seguintes categorias temáticas: o cotidiano dos escolares no processo de adesão a TARV e o silêncio na constituição de um cotidiano trágico.

\section{RESULTADOS E DISCUSSÃO}

\section{O cotidiano dos escolares no processo de adesão à TARV}

A cronicidade da aids implica em um processo de adesão a um regime medicamentoso completo e prolongado. Falhas na adesão aumentam o risco de incompleta supressão viral e de desenvolvimento de cepas virais resistentes aos medicamentos disponíveis. A adesão a um regime terapêutico requer mudanças no estilo de vida de indivíduos visando à realização de atividades específicas que promovam e mantenham a saúde. Essas atividades envolvem tomar as medicações prescritas regularmente, manter uma dieta adequada, automonitorar os sinais e sintomas da doença e submeter-se a avaliações de saúde periódicas. ${ }^{10}$

As crianças entrevistadas fazem uso de três drogas antirretrovirais. A apresentação da medicação, como informado pelos escolares, é oral. Os mesmos revelaram que a aceitação do medicamento na forma de comprimido é mais fácil que na apresentação líquida/suspensão, devido ao gosto do fármaco ser desagradável, conforme explicitam as falas a seguir.
Tomo comprimido e a seringa [suspensão] [...]. Eu prefiro comprimido, porque o líquido é amargo (Florzinha).

Os comprimidos que não queimam eu até mastigo (Tempestade).

Eu não gostava quando era líquido. É muito ruim, e o cheiro também, me dava vontade de botar tudo pra fora. O comprimido eu tomo, não tem problema não tem gosto não tem cheiro (Ben 10).

Sabe-se que a aceitação do medicamento na forma de comprimido é mais fácil que na apresentação líquida devido ao paladar desagradável que o medicamento proporciona, contudo, as indústrias farmacêuticas não produzem medicamento exclusivo para a faixa etária pediátrica. ${ }^{10}$

No entanto, diante da necessidade de ingerir uma medicação na forma líquida/suspensão, os escolares criaram estratégias capazes de "mascarar" o paladar do fármaco, fato que facilita a adesão à terapia antirretroviral, conforme as seguintes afirmações.

Tomo com café com leite, iogurte (Mulher Gavião).

Eu tomo com suco pra tirar o gosto (Batmam).

É fundamental o estabelecimento de uma aliança de cumplicidade entre a criança e o seu cuidador, onde as orientações medicamentosas devem ser compartilhadas entre o binômio para que ambos se sintam responsáveis pela administração da terapêutica implementada, conforme as falas a seguir.

A minha mãe leva o remédio [...] minha mãe me lembra (Mulher Gato).

Não me lembro muito de tomar não, mas meu pai me lembra. Mas às vezes eu não esqueço não porque minha vó também me lembra (Raven).

Minha mãe e minha vó me lembram, aí eu vou lá e tomo sozinha (Florzinha).

Tem vezes que eu tomo por mim mesmo, mas eles [pais e avó] que me lembram (Wanda).

O depoimento abaixo revela que o processo de adesão pode ser beneficiado quando se associa a ingestão medicamentosa às atividades rotineiras executadas no dia a dia.

[...] eu tomo de manhã quando eu acordo, quando eu vou pra escola [...] só que quando eu não vou pra escola eu tomo quando eu acordo, assim lá pra nove horas, dez horas mais ou menos [...] aí quando é de noitinha lá pra cinco horas da tarde eu tomo de novo (Wanda).

Ressalta-se que, embora atividades como dormir, acordar, realizar refeições facilitem a 
lembrança da ingestão dos ARV, a ausência de regularidade no horário das mesmas compromete a eficácia dos medicamentos. ${ }^{11}$

Os escolares costumam referir que não querem passar pelas experiências anteriores que caracterizavam um quadro clínico sintomático e, em função disso, relatam que procuram realizar corretamente o tratamento. Em pediatria um dos estímulos relacionados à adesão é a manutenção do bem estar físico, ou seja, as crianças apresentam o desejo de evitarem os momentos de hospitalização, conforme explicitam as falas a seguir.

Sei que é importante tomar o remédio [...] já fiquei internada e foi ruim [...] eu não quero ficar de novo! (Raven).

Sei que tem que tomar todo dia. Eu já fiquei internada e foi ruim (Florzinha).

É, porque se eu não tomar o remédio fico passando mal (Lanterna Verde).

Além do paladar, algumas características dos ARVs como sabor e cheiro são os fatores que mais dificultam a adesão. ${ }^{11}$ Deste modo, o maior entrave para adesão, encontrado a partir dos relatos dos escolares, foi a palatilidade.

O xarope [...] o gosto é ruim, queima a língua (Batman).

Mas o gosto é ruim tia, eu tomo quase vomitando. Eu boto a seringa lá no fundo da boca e tomo, é muito ruim tia, é horrível. O pior é o lamivudina que queima a boca (Tempestade).

Em um estudo realizado no interior paulista, com indivíduos que viviam com HIV/ aids, identificou-se que $14 \%$ dos portadores, quando questionados sobre os fatores que facilitam a adesão à terapêutica ARV, referiram o desaparecimento dos sintomas da doença. O mesmo também foi verificado neste estudo, uma vez que, sem sintomas, o portador pode retornar à sua vida normal, sem conseqüências para a vida social, e que a aids é, na atualidade, uma condição crônica com a qual todos podem conviver desde que haja a adesão adequada à terapêutica antirretroviral. ${ }^{12}$

Em contrapartida, a literatura aponta também que embora a ausência de sintomas e o fato de sentir-se bem sejam apontados como razões para adesão, a melhora dos sintomas em certos casos pode levar à interrupção do tratamento porque o indivíduo com HIV/aids avalia que não há necessidade da medicação, só retornando ao uso ao sentir-se mal. ${ }^{13}$

Neste contexto, observa-se que a linha que separa os aspectos que influenciam pacientes com aids na adesão e não-adesão à terapêutica antirretroviral é muito tênue, de modo que uma mesma situação pode constituir-se como facilitadora ou dificultadora do processo de adesão dependendo das circunstâncias.

Sabe-se que a baixa adesão constitui uma grande preocupação, pois alguns estudos identificaram elevado índice de não-adesão (em torno de $30 \%$ ), sendo que, nos casos de uso dos antiretrovirais a taxa de adesão recomendada gira em torno de $95 \%$, e ela inclui não simplesmente o uso, mas o uso regular, pois se isto não ocorre, pode haver falência no tratamento, possibilitando o aparecimento de novas cepas virais resistentes e comprometendo o prognóstico do indivíduo. ${ }^{11}$

Como entrave ao tratamento, a prática profissional tem revelado uma realidade que começou a ser desvelada entre os escolares do estudo, pois no decorrer da entrevista, mesmo aqueles que no início afirmaram tomar corretamente os medicamentos, revelaram que na prática cotidiana não era bem assim.

Eu tomo quase todo dia (Mulher Gavião).

Todo dia? Todo dia não (Raven).

Esqueço às vezes só... às vezes eu tomo antes e às vezes eu atraso só um pouquinho (Florzinha).

Todos os entrevistados afirmaram que já ficaram pelo menos um dia sem tomar as medicações. Os fatores responsáveis por tal fato são: palatilidade $(42,8 \%)$, esquecimento $(21,4 \%)$, administração da medicação em ambiente que não o domiciliar $(14,2 \%)$ e horário $(7,1 \%)$.

Observa-se que após a palatilidade os escolares não aderem à TARV por um motivo aparentemente "simples", que é o esquecimento de tomar os medicamentos. $\mathrm{O}$ depoimento abaixo retrata essa situação.

[...] porque eu chego, às vezes eu durmo e esqueço, e às vezes aí eu vou brincar e quando eu brinco eu esqueço de tudo, aí quando a minha vó me chama já são duas horas da tarde e não dá mais pra tomar porque tá atrasado! Meu maior problema é o esquecimento (Raven).

Entende-se que a complexidade do tratamento com o excessivo número de doses e drogas constituem fatores que contribuem para o esquecimento. Um estudo revelou que os pacientes esquecem a ingesta dos medicamentos principalmente durante os finais de semana pela dificuldade em cumprir os horários devido a compromissos sociais e que se sentem inibidos para tomá-los em um encontro ou festa. ${ }^{13}$ 
Foi observado também que a ingestão dos antirretrovirais em ambiente que não o domicílio, bem como o horário de administração dos mesmos constituíram-se em fatores dificultadores do processo de adesão.

Ah, eu não gosto de tomar duas vezes no dia [...] tomo só à noite [...], porque de manhã durmo, brinco aí eu esqueço (Raven).

O que eu não gosto no remédio é que tem que tomar de manhã... Aí eu não gosto. Quando eu tenho aula, eu to na escola e não tomo (Super Homem).

As atividades realizadas fora de casa são fatores que entrevêem na adesão uma vez que envolve mudanças na rotina, pois a presença de doses do meio do dia, estar muito ocupado, estar longe de casa ou sem os medicamentos no horário da dose, constituem dificuldades para "encaixar" o medicamento na rotina diária. ${ }^{14}$

\section{O silêncio na constituição de um cotidiano trágico}

Apesar do desenvolvimento científico e tecnológico que têm permitido o controle e evolução da aids, a cura continua como uma possibilidade distante. No contexto de uma condição crônica, a aids ainda desperta sentimento de preconceito, estigma, medo da morte, solidão, silêncio, entre outros. Assim, a aids quando presente na clientela pediátrica, desperta nos pais e/ou cuidadores questões emocionais frente à revelação do diagnóstico à criança HIV positiva. Sabe-se que o indivíduo com HIV/aids e sua família vivenciam a dicotomia entre tensões que podem anunciar o estado sorológico ou atitudes que objetivam esconder essa condição. ${ }^{15}$

Não conhecer a doença que apresenta foi uma realidade presente em seis dos dez escolares entrevistados na pesquisa. Tal fato chama a atenção para o seguinte questionamento: se a criança não sabe a doença que tem como é possível entender as reais necessidades de adesão à TARV?

Ao serem questionadas quanto à importância do medicamento, as crianças o associavam com a possibilidade de viver, de não permanecerem hospitalizados e de não adoecerem. No entanto, o ARV poderia ser considerado uma poderosa vitamina ou a vacina contra todas as doenças, pois os "super-heróis" não tinham conhecimento sobre o verdadeiro motivo da ingesta de tantos comprimidos.

Acho, acho importante pra não morrer (Mulher Gavião).
É... porque ajuda as pessoas, pra saúde ficar melhor também...é isso (Wanda).

Desta forma, começa-se a pensar que talvez os escolares não associem a ingestão dos antiretrovirais a sua rotina de atividades diárias por desconhecerem o real motivo para a tomada do medicamento: viver com aids. $\mathrm{O}$ seguinte relato confirma essa situação:

[...] de manhã eu tomo café e vou pra escola, eu acordo às seis horas da manhã. Vou pra escola, aí na escola eu como, tomo café eu brinco no recreio, ai quando eu volto, eu vejo desenho e Chaves [...] e às vezes eu durmo e quando não durmo fico brincando [...] (Raven).

Acredita-se que o silêncio em relação à infecção compromete o estabelecimento de redes de apoio quando há a necessidade de ações de educação em saúde para orientar esses escolares sobre a importância da adesão, bem como os riscos de desenvolvimento de infecções oportunistas e Doenças Sexualmente Transmissíveis (DSTs).

A aids é uma condição crônica e seu controle tem constituído um desafio aos profissionais de saúde na medida em que seu tratamento envolve a participação ativa dos pacientes no sentido de modificar alguns comportamentos prejudiciais à sua própria saúde e assimilar outros que beneficiem sua condição clínica. Assim, quando a criança desconhece sua realidade de portador de uma condição crônica como a aids, ela ignora a importância de introdução o ARV em sua rotina, não desenvolvendo o autocuidado consigo e com os demais.

A partir do exposto vale questionar-se sobre até que ponto o silenciamento a respeito da doença pelos cuidadores aos escolares começa a ser uma questão de negligência que pode colocar em risco a saúde de outras pessoas, e se este escolar desconhece ter uma doença sexualmente transmissível será que o mesmo adotará medidas de barreira durante as relações sexuais.

Ainda que pais/cuidadores e as crianças sobrevivam a um cotidiano marcado pela necessidade do ocultamento, vivenciando a possibilidade de estigmatização e preconceito, cabe a equipe interdisciplinar pensar junto com essa família a melhor maneira de revelação do status sorológico e trazer à tona os riscos e benefícios da conscientização de ter a aids.

Vivenciar uma situação de doença sem saber sua real condição constitui um paradoxo, como revelado no depoimento a seguir: 
[...] minha mãe falou pra eu tomar porque tem dois bichinhos brigando na minha barriga [...] ai tem o meu que dá força pra me alimentar e o remédio que dá força pro meu. E tem o outro [bichinho] que briga com o meu. Aí eu tenho que tomar o remédio pra fortalecer o meu e matar os outros. Aí se eu matar os outros, se eu ficar tomando assim um monte de dia, assim certinho, e matar o outro, eu posso até parar de tomar o remédio (Lanterna Verde).

Entende-se que embora as crianças sejam vistas como vítimas da epidemia, uma vez que foram contaminadas por transmissão vertical, quando a sociedade toma conhecimento da presença delas em escolas, parques e outros, assumem uma postura de medo, isolando-as do convívio com as demais.

Em virtude deste fato, por receio do estigma e da discriminação, os cuidadores optam por não revelarem o diagnóstico, embora criem estratégias de enfrentamento para promover a adesão à terapia medicamentosa. Desta forma, o cuidador conta com a cumplicidade do profissional de saúde ao recorrerem à utilização de metáforas para estabelecer uma comunicação e promover a adesão junto à criança. Tal atitude vai a confronto com o conceito de adesão uma vez que mantém a criança na condição de coadjuvante de sua saúde. ${ }^{15}$

Ao dar vez e voz às crianças torna-se possível compreender a dimensão da aids em suas vidas em função do tratamento, dos retornos ambulatoriais, exames e frequentes hospitalizações. A criança percebe a gravidade de sua doença, conhece o tratamento e os efeitos colaterais de alguns medicamentos, procura entender e justificar os procedimentos realizados, assim como o desconforto das rotinas em função de sua recuperação e da cura de algo que lhes é velado.

\section{CONSIDERAÇÕES FINAIS}

Entende-se que a adesão ocorre quando o indivíduo apropria-se do tratamento, ou seja, quando há um comprometimento decorrente da compreensão do real significado da terapêutica e sua eficácia.

O presente estudo permitiu identificar alguns elementos facilitadores do processo de adesão, a saber: apresentação do fármaco na forma de comprimidos; elaboração de estratégias pelos próprios escolares com a finalidade de mascarar o sabor desagradável dos medicamentos; parceria entre cuidador e escolar, bem como a ausência de sinais e sintomas na atualidade e o desejo de manutenção do estado de saúde, tendo em vista as lembranças relacionadas à fase agudizada da doença e a necessidade de internação hospitalar.

Verifica-se vários fatores que contribuem para a adesão ou a não-adesão à TARV, variando desde o sigilo do diagnóstico até as dificuldades com a ingestão dos medicamentos. Percebe-se que as crianças têm dificuldades para cumprir os horários das medicações, seja pela inserção em atividades típicas de sua idade (como escola e brincadeiras), pela forma de apresentação do fármaco (sabor, tamanho e odor), ou pela necessidade de ingerir um medicamento sem que conheça o motivo.

Assim, no que se tange à assistência do enfermeiro, como participante da equipe multiprofissional, com vistas a aumentar o processo de adesão, sabe-se que a grande aliada para a adesão é a assistência individualizada, na qual a relação enfermeira-cuidador-criança deve ser permeada por confiança, empatia, respeito, privacidade e atitudes positivas.

Desta forma, o enfermeiro deve ajudar tanto os pais/cuidadores quanto os escolares a se tornarem ativos partícipes no que concerne às questões relacionadas à doença e ao tratamento. Para tanto, basta dar a criança à oportunidade de participar das tomadas de decisão, desenvolvendo, gradualmente, a autonomia e o autocuidado.

É necessário que os profissionais de saúde reconheçam que a não adesão é um fenômeno que deve ser trabalhado junto à criança e que é importante à implementação de estratégias capazes de incentivar a adesão ao tratamento, como estudo de casos, oficinas interdisciplinares, grupos de adesão, com o objetivo de acolher o escolar e o seu cuidador, na perspectiva de fazer mudar sua condição, levando-o a se tornar sujeito do processo. Assim, cabe ao enfermeiro atuar junto ao binômio cuidador-criança, para proporciona-lhes melhor qualidade de vida durante esse tratamento que é tão oneroso e desgastante para ambos.

\section{REFERÊNCIAS}

1. Ministério da Saúde (BR). Programa Nacional de DST/aids. Boletim epidemiológico. Brasília (DF): MS; 2010.

2. Silva RAR, Rocha VM, Davim RMB, Torres GV. Formas de enfrentamento da AIDS: opinião de mães de crianças soropositivas. Rev Latino-am Enfermagem [online]. 2008 Apr [acesso 2010 Jan 13]; 16(2):260-5. Disponível em: http:/ / www. scielo.br/scielo.php?script=sci_arttext\&pid=S0104$11692008000200014 \& \operatorname{lng}=\mathrm{en} \& \mathrm{nrm}=\mathrm{iso} \& \mathrm{t} \operatorname{lng}=\mathrm{pt}$ 
3. Guerra CPP, Seidl EMF. Crianças e adolescentes com HIV/aids: revisão de estudos sobre revelação do diagnóstico, adesão e estigma. Paidéia [online]. 2009 Jan [acesso 2010 Jan 13]; 19(42):59-65. Disponível em: http://www.scielo.br/scielo.php?script=sci_ arttext\&pid=S0103-863X2009000100008

4. Ministério da Saúde (BR). Adesão aos anti-retrovirais: manual para profissionais de saúde. Brasília (DF): Unidade de Assistência e Tratamento; 2005.

5. Paula CC, Crossetti MGO. O modo de cuidar no encontro com o ser-criança que convive com aids: o experienciar da finitude e a ética. Texto Contexto Enferm [online]. 2005 Abr-Jun [acesso 2010 Jan 04]; 14(2):193-201. Disponível em: http:/ / www. scielo.br/scielo.php?script=sci_arttext\&pid=S010407072005000200006\&lng=pt4

6. Whaley LF, Wong DL. Enfermagem pediátrica: elementos essenciais à intervenção efetiva. $5^{\mathrm{a}}$ ed. Rio de Janeiro (RJ): Guanabara Koogan; 1999.

7. Minayo MCS. O desafio do conhecimento: pesquisa qualitativa em saúde. $8^{a}$ ed. São Paulo (SP): Hucitec; 2004.

8. Coa TF, Pettengill MAM. Autonomia da criança hospitalizada frente aos procedimentos: crenças da enfermeira pediatra. Acta Paul Enferm [online]. 2006 Dez [acesso 2010 Jan 04]; 19(4):433-8. Disponível em: http://www.scielo.br/scielo.php?script=sci_ arttext\&pid=S0103-21002006000400011\&lng $=p t$

9. Bardin L. Análise de conteúdo. Lisboa (PT): Edições 70; 1979.

10. Feitosa AC, Andrade LM, Lima HJA, Beserra EP, Caetano JA. Terapia anti-retroviral: fatores que interferem na adesão de crianças com HIV/aids. Esc Anna Nery Rev Enferm [online]. 2008 Set [acesso 2010 Jan 04]; 12(3):515-21. Disponível em: http://www.eean.ufrj.br/revista_enf/20083/ artigo\%2016.pdf

11. Silva ALCN, Waidman MAP, Marcon SS. Adesão e não-adesão à terapia antirretroviral: as duas faces de uma mesma vivência. Rev Bras Enferm [online]. 2009 Apr [citado 2010 Jan 13]; 62(2):213-20. Disponível em: http:/ / www.scielo.br/scielo.php?pid=S003471672009000200007\&script=sci_arttext

12. Gir E, Vaichulonis CG, Oliveira MD. Adesão à terapêutica antirretroviral por indivíduos com HIV/AIDS assistidos em uma instituição do interior paulista. Rev Latino-am Enfermagem [online]. 2005 Out [acesso 2010 Jan 13]; 13(5):634-41. Disponível em: http://www.scielo.br/scielo.php?script=sci_a rttext\&pid=S0104-11692005000500005

13. Colombrini MRC, Lopes MHBM, Figueiredo RM. Adesão à terapia antirretroviral para HIV/ AIDS. Rev Esc Enferm USP [online]. 2006 Dez [acesso 2010 Jan 13]; 40(4):576-81. Disponível em: http:/ / www.scielo.br/ scielo.php?pid=S008062342006000400018\&script=sci_arttext

14. Kourrouski MFC. Adesão ao tratamento: vivências de adolescentes com HIV/aids [dissertação]. Ribeirão Preto (SP): Universidade de São Paulo. Escola de Enfermagem de Ribeirão Preto; 2008.

15. Gomes AMT. Silêncio, silenciamento e ocultamento na terapia antirretroviral: desvelando o discurso de cuidadores de crianças [tese]. Rio de Janeiro (RJ): Universidade Federal do Rio de Janeiro. Escola de Enfermagem Anna Nery; 2005. 